\title{
The Impact of Proper PPE Protocol in A Highly Community Spread of Infectious COVID-19 in Semporna
}

\author{
Muhammad Farid Bin Zainal Abidin ${ }^{1}$, Nur Farhana Binti Zainan ${ }^{1}$, Mafeitzeral \\ Bin Mamat ${ }^{2}$, Sivasankar Pubalan ${ }^{1}$, Mohd Syahfadzreen Bin Yunus ${ }^{1}$ \\ ${ }^{1}$ Hospital Semporna, Sabah, Malaysia; ${ }^{2}$ Gleneagles Medini, Johor, Malaysia \\ DOI: http://dx.doi.org/10.31344/ijhhs.v5i0.312
}

Introduction: The district of Semporna, Sabah was majorly hit by the third wave surge of COVID-19 in September 2020. At the peak of the crisis, services in Semporna Hospital were paralyzed with 40 healthcare workers (HCW) found to be COVID-19 positive; contributing to $14 \%$ of the total manpower.

Objectives: Due to the immediate crisis, the main priority was to control the spread of COVID19 amongst the Semporna Hospital HCWs. This was important to curb direct infection from handling suspected patients seeking treatment in Semporna Hospital. Based on the hospital capabilities and resources, a comprehensive modified protocol was needed to control the situation.

Methods: Semporna Hospital pandemic emergency task force was established with the presence of multi department and specialties. Multiple issues were raised and attended to, especially; issue of preparedness, low PPE stock and Emergency Department infrastructure.

Results: The number of Semporna Hospital HCWs infected with COVID-19 was minimal after the initial disaster. Emergency Department infrastructure was improvised, workflow processes modified, HCW protection education prioritized and complete PPE sets were stocked up. All these efforts were under the strict supervision of the infectious control unit. The presence of multiple units of Powered Air-Purifying Respirator (PAPR) completed our adherence to the Ministry of Health $(\mathrm{MOH})$ guidelines in managing the highly infectious level 4 patients for aerosolized generating procedures (AGP).

Conclusion: This achievement can be used as a preparedness reference for other non-specialist district hospitals in Malaysia.

Keywords: PPE, PAPR, Donning and doffing 